\section{DDR2 blockade might be a good treatment option for patients with OA}

Osteoarthritis $(\mathrm{OA})$ is characterized by proteoglycan loss, degradation of type II collagen and subsequent destruction of articular cartilage matrix. Animal experiments have provided strong evidence for the involvement of a tyrosine kinase receptor for type II collagen, discoidin domain receptor 2 (DDR2), in the pathogenesis of OA.

Sunk et al. analyzed human osteoarthritic articular cartilage obtained from 16 patients (mean age 64.8 years) for expression of DDR2 and matrix metalloproteinase 13 (MMP13), because DDR2 expression and activation has previously been shown to result in increased synthesis of this MMP. Levels of DDR2 and MMP13 were found to directly correlate with the severity of cartilage damage.

Chondrocytes were obtained from the articular cartilage samples and cultured for 24 hours. Exposure to type II collagen resulted in increased expression of DDR2 and MMP13. The authors hypothesize that this process might be integral to the initiation of type II collagen degradation in early $\mathrm{OA}$, since disruption of the pericellular microenvironment could expose collagen type II fibrils to chondrocytes.

Blockade of DDR2 is, therefore, a potential therapeutic option for patients with early $O A$.

Original article Sunk I-G et al. (2007) Increased expression of discoidin domain receptor 2 is linked to the degree of cartilage damage in human knee joints. Arthritis Rheum 56: 3685-3692

\section{Adrenomedullin is associated with hypertension in patients with systemic sclerosis}

Adrenomedullin is a small peptide involved in several physiological processes, including angiogenesis, growth regulation and vasodilation. Evidence suggests that it also has anti-inflammatory effects, and has been observed at elevated plasma levels in patients with autoimmune diseases such as rheumatoid arthritis. Mok and colleagues investigated the association between adrenomedullin levels and vascular manifestations in a cohort of patients with systemic sclerosis (SSc).

The study population included 62 consecutive patients (mean age 52.1 years, 58 female) with either diffuse $(n=14)$ or limited $(n=48)$ SSc and 21 healthy control subjects. Participants were examined for the presence of Reynaud's phenomenon (RP) and elevated pulmonary artery pressure (PAP), and adrenomedullin levels were measured using radioimmunoassay.

Overall, levels of adrenomedullin in patients with SSc were not significantly different from controls. In patients with elevated PAP (15 [24.2\%] of 62 patients), adrenomedullin levels were significantly higher than in patients without pulmonary hypertension and control subjects. SSc patients with RP (present in $91.1 \%$ of patients, with severe RP present in $38.7 \%$ ) had similar levels of adrenomedullin compared with those without RP and controls; however, patients with both elevated PAP and presence of RP had significantly higher adrenomedullin levels than patients with neither RP nor elevated PAP and patients with RP alone.

The authors conclude that pulmonary hypertension might be associated with elevated adrenomedullin levels in patients with SSc. Further studies will be necessary to investigate the source and role of adrenomedullin in relation to endothelin-1, hypertension, RP and prognosis of SSC.

Original article Mok MY et al. (2007) Elevated plasma adrenomedullin and vascular manifestations in patients with systemic sclerosis. J Rheumatol 34: 2224-2229

\section{Dual-energy X-ray absorptiometry is a highly sensitive measure of bone loss in early RA}

Radiographic joint damage, usually assessed with the Sharp or Larsen scoring systems, is the only established outcome measure for evaluating inflammation-related bone damage in patients with rheumatoid arthritis (RA). The sensitivity of this technique, however, is questionable; detectable joint erosion is usually present only in established disease rather than in early RA. Haugeberg and colleagues from Leeds University, UK, therefore, examined the use of dual-energy X-ray absorptiometry (DEXA) as an alternative approach to measuring bone involvement in patients with early RA.

The study population included 79 patients with early RA (mean age 49.7 years, mean disease duration 8.5 months) and poor prognosis. DEXA was used to measure hand bone mineral density (BMD) at baseline, 24 weeks 